\title{
Energy Cost of Free-Play Activities in 10- to 11-Year-Old Children
}

\author{
Kelly A. Mackintosh, Kate Ridley, Gareth Stratton, and Nicola D. Ridgers
}

\begin{abstract}
Objective: This study sought to ascertain the energy expenditure (EE) associated with different sedentary and physically active free-play activities in primary school-aged children. Methods: Twenty-eight children (13 boys; $11.4 \pm 0.3$ years; $1.45 \pm 0.09$ $\mathrm{m} ; 20.0 \pm 4.7 \mathrm{~kg} \cdot \mathrm{m}^{-2}$ ) from 1 primary school in Northwest England engaged in 6 activities representative of children's play for 10 minutes (drawing, watching a DVD, playground games and free-choice) and 5 minutes (self-paced walking and jogging), with 5 minutes rest between each activity. Gas exchange variables were measured throughout. Resting energy expenditure was measured during 15 minutes of supine rest. Results: Child (Schofield-predicted) MET values for watching a DVD, self-paced jogging and playing reaction ball were significantly higher for girls $(P<.05)$. Conclusion: Utilizing a field-based protocol to examine children's free-living behaviors, these data contribute to the scarcity of information concerning children's EE during play to update the Compendium of Energy Expenditures for Youth.
\end{abstract}

Keywords: youth, physical activity, measurement

In 2008, Ridley and colleagues ${ }^{1}$ developed the Compendium of Energy Expenditures for Youth to enable researchers to understand the energy costs associated with commonly performed activities during daily living. However, two-thirds of the information listed was estimated using the adult compendium. ${ }^{2}$ The compendium should be based on measured child-specific energy costs during both structured and unstructured activities across the intensity spectrum (ie, sedentary, light, moderate, vigorous). However, the majority of previous research used highly structured protocol's to assess children's energy expenditure (EE) to standardize movement patterns and facilitate interindividual comparisons. Such laboratorybased protocols may be limited in the assessment of free-living behaviors. ${ }^{3}$ For example, the energy cost of predetermined treadmill and/or running speeds are often examined, yet may have little ecological validity as these behaviors are self-paced by individuals and performed over-ground during daily living. In addition, given the sporadic and intermittent nature of children's physical activity (PA) there is a need to establish children's EE during play, with greater autonomy over mode, duration and intensity, which is more representative of their free-living behaviors. Unstructured free-play and playground games are examples of common behaviors children engage in, both at school and after school, ${ }^{4}$ yet data on active freeplay that informed the 2008 compendium were scarce. Indeed, the "unstructured outdoor play" MET value was based on 1 study that measured school quadrangle play in the 1920s.

The aim of this study was to ascertain the EE associated with different sedentary and physically active free-play activities in children.

Mackintosh is with the College of Engineering, Swansea University, United Kingdom. Ridley is with the Sport, Health, and Physical Education (SHAPE) Research Centre, Flinders University, Australia. Stratton is with the Applied Sports Technology Exercise and Medicine Research Centre, Swansea University, United Kingdom. Ridgers (nicky.ridgers@ deakin. edu.au) is with the Institute for Physical Activity and Nutrition, Deakin University, Australia.

\section{Methods}

\section{Study Participants}

Twenty-eight 10- to 11-year-old children (13 boys, 15 girls) from 1 primary school in Northwest England participated in the study. Descriptive characteristics are shown in Table 1. Participants provided written assent and the primary caregiver provided informed written consent. The study was approved by the University Ethics Committee.

\section{Activities}

Resting energy expenditure (REE) was measured during 15 minutes of supine rest in a quiet, dimly lit room after at least 1 hour of fasting. Children subsequently took part in 6 different activities chosen to represent intermittent and continuous behaviors undertaken in freeplay situations both on their own and with other people. The activities took place either in the classroom or in the school playground in a randomized order, with 5 minutes of seated rest between each activity. The 6 free-play activities that children participated in were

1. Drawing/coloring: The child sat at a classroom table and was provided with materials to draw/color pictures on their own

2. DVD watching: The child sat inside the classroom and watched a DVD on their own

3. Self-paced brisk walking: The child briskly walked around a marked circular track on the playground at a self-selected pace

4. Self-paced jogging: The child jogged around the marked circular track at a self-selected pace

5. Playground games: The child played 3 different games in a standardized order (hopscotch, Frisbee, and reaction ball) for 3.3 minutes each on the playground with a researcher, with no breaks in between

6. Free choice games: The child was provided with the opportunity to play different games on their own or with a researcher. 
Table 1 Descriptive Characteristics of Participants (Mean \pm SD)

\begin{tabular}{lcccccc}
\hline & $\mathbf{n}$ & Age $(\mathbf{y r})$ & Stature $(\mathbf{m})$ & Body mass $\mathbf{( k g})$ & BMI $\mathbf{( k g \cdot \mathbf { m } ^ { - 2 } )}$ & BMI-z \\
\hline Total & 28 & $11.4 \pm 0.3$ & $1.45 \pm 0.09$ & $42.4 \pm 9.9$ & $20.0 \pm 4.7$ & $0.68 \pm 0.9$ \\
Girls & 15 & $11.4 \pm 0.3$ & $1.44 \pm 0.10$ & $39.7 \pm 6.7$ & $19.5 \pm 5.6$ & $0.28 \pm 1.0$ \\
Boys & 13 & $11.4 \pm 0.3$ & $1.47 \pm 0.08$ & $45.5 \pm 12.2$ & $20.6 \pm 3.4$ & $1.00 \pm 0.8$ \\
\hline
\end{tabular}

A Frisbee, soccer ball, tennis racquets and balls, skipping ropes, hula hoops, reaction ball, and a large dice were provided to facilitate the games. The child was able to freely change the game(s) played during this time.

All activities were 10 minutes in duration, with the exception of self-paced brisk walking and self-paced jogging which were 5 minutes in length. This was to ensure that children could sustain the self-selected pace for the whole activity duration.

\section{Outcome Measures}

EE was assessed using a portable, open-circuit indirect calorimetry system (MetaMax 3B, Cortex, Leipzig, Germany) that measured breath-by-breath oxygen consumption $\left(\mathrm{VO}_{2}\right)$. The MetaMax was calibrated before each trial using the manufacturer recommended procedure. Respiratory volume was calibrated using a 3-L syringe. Gas sensors were calibrated against known concentrations of gases (16\% oxygen, $4 \%$ carbon dioxide). The MetaMax analyzer unit was attached to the child around their upper body using a pediatric harness with adjustable Velcro straps. A facemask was secured over the child's nose and mouth using an adjustable nylon harness. A bidirectional digital turbine flowmeter was inserted into the facemask to measure the volume of inspired and expired air. A sample line connecting the turbine and analyzer unit to determine the content of oxygen and carbon dioxide. Data were retrieved at the end of each trial for analysis by manufacturer proprietary software (MetaMax, version 2.4, Statera Edition). Data were reduced to 10 second epochs for analysis due to the free-play nature of the activities being performed.

REE was calculated by removing the first and last 5 minutes and averaging the remaining data during the resting phase. For each activity $\mathrm{VO}_{2}\left(1 \cdot \mathrm{min}^{-1}\right)$, relative $\mathrm{VO}_{2}\left(\mathrm{ml} \cdot \mathrm{kg}^{-1} \cdot \mathrm{min}^{-1}\right)$, and EE $\left(\mathrm{J} \cdot \mathrm{kg}^{-1} \cdot \mathrm{min}^{-1}\right)$ were calculated by removing the first 2.5 minutes and last 0.5 minute and averaging the remaining data. As children can achieve steady state in 2 to 3 minutes, ${ }^{5}$ the individual playground games (hopscotch, Frisbee and reaction ball) were also examined separately. For these activities, the first 2.5 minutes were removed and an average was taken across 50 seconds. After 2.5 minutes, EE values had reached steady state, which was indicated by a plateau in $\mathrm{VO}_{2}$ and $\mathrm{VCO}_{2}$ where values varied by less than $15 \% . \mathrm{VO}_{2}$ was converted to EE using the values of $1 \mathrm{~L} \mathrm{O}_{2}=4.9 \mathrm{kcal} .{ }^{6} \mathrm{All}$ participants were assessed with the same calorimeter.

An estimate of daily RMR was calculated for each participant using the sex-, age-, and mass-specific Schofield prediction equations. ${ }^{7}$ Child metabolic equivalents (Child METs) were calculated by dividing activity EE by predicted RMR.?

\section{Statistical Analyses}

All data are expressed as means and standard deviations. One-way analyses of variance were conducted to examine sex differences for the descriptive characteristics and the energy cost of the freeplay activities. All statistical analyses were conducted using PASW
Statistics 22 (SPSS, Chicago, Il). Statistical significance was set at $P<.05$.

\section{Results}

Technical issues with the MetaMax, resulted in 3 children's data (1 boy, 2 girls) being fully lost. Incomplete data were recorded for 8 children ( 2 boys, 6 girls). All collected data were analyzed for each activity. The energy cost of the sedentary and active free-play behaviors, along with the sample sizes for each behavior, are shown in Table 2 and Table 3, respectively.

There were no significant sex differences for any of the descriptive characteristics. Self-paced walking and jogging speeds were $1.71 \pm 0.1 \mathrm{~m} \cdot \mathrm{s}^{-1}$ (range 1.31 to $2.00 \mathrm{~m} \cdot \mathrm{s}^{-1}$ ) and $2.59 \pm 0.32 \mathrm{~m} \cdot \mathrm{s}^{-1}$, (range 1.31 to $2.00 \mathrm{~m} \cdot \mathrm{s}^{-1}$ ) for the whole sample, respectively. Specifically, boys and girls speeds were $1.65 \pm 0.2 \mathrm{~m} \cdot \mathrm{s}^{-1}\left(1.31\right.$ to $\left.2.00 \mathrm{~m} \cdot \mathrm{s}^{-1}\right)$ and $1.86 \pm 0.1 \mathrm{~m} \cdot \mathrm{s}^{-1}\left(1.55\right.$ to $\left.1.97 \mathrm{~m} \cdot \mathrm{s}^{-1}\right)$ for walking, and $2.5 \pm 0.4$ $\mathrm{m} \cdot \mathrm{s}^{-1}\left(1.91\right.$ to $\left.2.96 \mathrm{~m} \cdot \mathrm{s}^{-1}\right)$ and $2.67 \pm 0.3 \mathrm{~m} \cdot \mathrm{s}^{-1}\left(2.19\right.$ to $\left.3.3 \mathrm{~m} \cdot \mathrm{s}^{-1}\right)$ for jogging, respectively. In line with the adult compendium, the energy cost associated with various speed ranges (1.31 to 1.80 $\mathrm{m} \cdot \mathrm{s}^{-1}, 1.81$ to $2.30 \mathrm{~m} \cdot \mathrm{s}^{-1}, 2.31$ to $2.80 \mathrm{~m} \cdot \mathrm{s}^{-1}$ and 2.81 to $3.30 \mathrm{~m} \cdot \mathrm{s}^{-1}$ ) are shown in Table 3. Girls expended more energy than boys for drawing $(P<.05)$. Child MET values for watching a DVD, selfpaced jogging and playing reaction ball were significantly higher for girls $(P<.05)$.

\section{Discussion}

The activity that was least consistent with the 2008 compendium was self-selected brisk walking, which has been used to describe moderate-intensity PA (MPA) in public health recommendations. On average, children walked at $1.7 \mathrm{~m} \cdot \mathrm{s}^{-1}$, which was $0.2 \mathrm{~m} \cdot \mathrm{s}^{-1}$ faster than the identified 'hard' speed, ${ }^{1}$ and elicited an EE of $\geq 4$ METs. Notably, 4 METs is increasingly used to quantify children's MPA in accelerometry studies, ${ }^{8}$ which this study supports. For jogging, children self-selected a speed that was consistent with moderate running in the compendium, though the energy cost was closer to the MET value identified for light jogging. ${ }^{1}$ Despite this discrepancy, self-paced jogging exceeded the vigorous-intensity PA (VPA) threshold of 6 METs, which is typically used to quantify children's VPA in accelerometry studies. ${ }^{8}$

Little data were available to identify the energy cost of unstructured outdoor play in the original compendium. ${ }^{1}$ Using a protocol where children had autonomy over the intensity they engaged in to play the different playground games, which were sporadic and intermittent in nature, the energy cost was consistent with the original 5 METs value. This is a positive finding and further supports the inclusion of active play as an example of PA in current recommendations. ${ }^{9}$ One of the playground games children played during this condition was Frisbee, which was an activity where the energy cost was estimated using adult data. ${ }^{2}$ This study provides new information about the energy cost of this activity during unstructured 


\begin{tabular}{|c|c|c|c|c|c|c|c|c|c|}
\hline & \multirow[b]{2}{*}{$\mathbf{n}$} & \multicolumn{2}{|c|}{$\mathrm{O}_{2}$ uptake $\left(\mathrm{ml} \cdot \mathrm{kg}^{-1} \cdot \mathrm{min}^{-1}\right)$} & \multicolumn{2}{|c|}{$\mathrm{O}_{2}$ uptake $\left(\mathrm{I} \cdot \mathrm{min}^{-1}\right)$} & \multicolumn{2}{|c|}{$\mathrm{EE}\left(\mathrm{kcal} \cdot \mathrm{min}^{-1}\right)$} & \multicolumn{2}{|c|}{ EE (Child METs) } \\
\hline & & Mean \pm SD & Min-Max & Mean \pm SD & Min-Max & Mean \pm SD & Min-Max & Mean \pm SD & Min-Max \\
\hline \multicolumn{10}{|l|}{ Rest } \\
\hline Girls & 10 & $4.6 \pm 0.9$ & $3.5-6.3$ & $0.20 \pm 0.04$ & $0.13-0.26$ & $0.96 \pm 0.18$ & $0.63-1.26$ & $1.1 \pm 0.2$ & $0.8-1.5$ \\
\hline Boys & 12 & $5.0 \pm 2.3$ & $1.6-9.7$ & $0.21 \pm 0.07$ & $0.07-0.31$ & $1.03 \pm 0.37$ & $0.33-1.54$ & $1.0 \pm 0.4$ & $0.3-1.7$ \\
\hline Combined & 22 & $4.8 \pm 1.8$ & $1.6-9.7$ & $0.20 \pm 0.06$ & $0.07-0.31$ & $0.99 \pm 0.29$ & $0.33-1.54$ & $1.1 \pm 0.3$ & $0.3-1.7$ \\
\hline \multicolumn{10}{|l|}{ Drawing } \\
\hline Girls & 12 & $6.8 \pm 1.6$ & $4.2-9.8$ & $0.27 \pm 0.05$ & $0.22-0.37$ & $1.34 \pm 0.24$ & $1.06-1.82$ & $1.6 \pm 0.3$ & $1.1-2.1$ \\
\hline Boys & 10 & $5.1 \pm 1.3$ & $2.9-7.1$ & $0.21 \pm 0.05$ & $0.13-0.31$ & $1.03 \pm 0.25$ & $0.62-1.54$ & $1.1 \pm 0.2$ & $0.6-1.4$ \\
\hline Combined & 22 & $6.0 \pm 1.7$ & $2.9-9.8$ & $0.25 \pm 0.06$ & $0.13-0.37$ & $1.20 \pm 0.28$ & $0.62-1.82$ & $1.3 \pm 0.4$ & $0.6-2.1$ \\
\hline \multicolumn{10}{|c|}{ DVD watching } \\
\hline Girls & 12 & $5.0 \pm 1.3$ & $3.1-7.1$ & $0.20 \pm 0.04$ & $0.16-0.29$ & $0.99 \pm 0.20$ & $0.78-1.44$ & $1.2 \pm 0.3$ & $0.8-1.7$ \\
\hline Boys & 10 & $4.2 \pm 2.1$ & $2.0-9.0$ & $0.17 \pm 0.05$ & $0.09-0.26$ & $0.83 \pm 0.26$ & $0.42-1.25$ & $0.9 \pm 0.3$ & $0.4-1.6$ \\
\hline Combined & 22 & $4.7 \pm 1.7$ & $2.0-9.0$ & $0.19 \pm 0.05$ & $0.09-0.29$ & $0.91 \pm 0.24$ & $0.42-1.44$ & $1.0 \pm 0.3$ & $0.4-1.7$ \\
\hline
\end{tabular}

Note. $\mathrm{ml} \cdot \mathrm{kg}^{-1} \cdot \mathrm{min}^{-1}=$ milliliters (of oxygen) per kilogram body weight; $1 \cdot \mathrm{min}^{-1}=$ liters (of oxygen) per minute.

Table 3 Energy Expenditure of Active Free-Play Behaviors

\begin{tabular}{|c|c|c|c|c|c|c|c|c|c|}
\hline & \multirow[b]{2}{*}{$\mathbf{n}$} & \multicolumn{2}{|c|}{ O uptake $\left(\mathrm{ml} \cdot \mathrm{kg}^{-1} \cdot \mathrm{min}^{-1}\right)$} & \multicolumn{2}{|c|}{$\mathrm{O}_{2}$ uptake $\left(\mathrm{I} \cdot \mathrm{min}^{-1}\right)$} & \multicolumn{2}{|c|}{$\mathrm{EE}\left(\mathrm{kcal} \cdot \mathrm{min}^{-1}\right)$} & \multicolumn{2}{|c|}{ EE (Child METs) } \\
\hline & & Mean \pm SD & Min-Max & Mean \pm SD & Min-Max & Mean \pm SD & Min-Max & Mean \pm SD & Min-Max \\
\hline \multicolumn{10}{|c|}{ Self-paced walking } \\
\hline Girls & 10 & $20.1 \pm 3.0$ & $14.9-23.7$ & $0.82 \pm 0.11$ & $0.66-0.97$ & $4.04 \pm 0.55$ & $3.23-4.76$ & $4.8 \pm 0.6$ & $3.7-5.5$ \\
\hline Boys & 11 & $17.8 \pm 5.1$ & $11.3-27.0$ & $0.79 \pm 0.25$ & $0.43-0.25$ & $3.86 \pm 1.22$ & $2.11-6.13$ & $3.9 \pm 1.0$ & $2.3-5.5$ \\
\hline Combined & 21 & $18.9 \pm 4.3$ & $11.3-27.0$ & $0.81 \pm 0.19$ & $0.43-1.25$ & $3.95 \pm 0.94$ & $2.11-6.13$ & $4.3 \pm 0.9$ & $2.3-5.5$ \\
\hline \multicolumn{10}{|c|}{ Self-paced jogging } \\
\hline Girls & 9 & $32.8 \pm 5.1$ & $27.1-42.0$ & $1.32 \pm 0.21$ & $0.89-1.60$ & $6.46 \pm 1.05$ & $4.35-7.82$ & $7.7 \pm 1.1$ & $6.0-9.2$ \\
\hline Boys & 11 & $33.2 \pm 6.6$ & $19.3-44.7$ & $1.48 \pm 0.45$ & $0.83-2.32$ & $7.25 \pm 2.19$ & $4.08-11.35$ & $7.2 \pm 1.4$ & $4.2-9.1$ \\
\hline Combined & 20 & $33.0 \pm 5.81$ & $19.3-44.7$ & $1.41 \pm 0.36$ & $0.83-2.32$ & $6.89 \pm 1.77$ & $4.08-11.35$ & $7.4 \pm 1.2$ & $4.2-9.2$ \\
\hline \multicolumn{10}{|c|}{$1.31-1.80 \mathrm{~m} \cdot \mathrm{s}^{-1}$} \\
\hline Girls & 6 & $15.1 \pm 2.9$ & $11.3-18.4$ & $0.70 \pm 0.22$ & $0.43-1.03$ & $3.45 \pm 1.10$ & $2.11-5.03$ & $3.4 \pm 0.7$ & $2.3-4.2$ \\
\hline Boys & 8 & $19.3 \pm 3.2$ & $14.9-23.7$ & $0.78 \pm 0.10$ & $0.66-0.97$ & $3.81 \pm 0.50$ & $3.23-4.76$ & $4.5 \pm 0.6$ & $3.7-5.5$ \\
\hline Combined & 14 & $17.2 \pm 3.6$ & $11.3-23.7$ & $0.74 \pm 0.17$ & $0.43-1.03$ & $3.63 \pm 0.84$ & $2.11-5.03$ & $4.0 \pm 0.9$ & $2.3-5.5$ \\
\hline \multicolumn{10}{|c|}{$1.81-2.30 \mathrm{~m} \cdot \mathrm{s}^{-1}$} \\
\hline Girls & 4 & $30.3 \pm 4.6$ & $26.0-36.6$ & $1.36 \pm 0.49$ & $0.74-2.18$ & $6.67 \pm 2.41$ & $3.64-10.70$ & $6.6 \pm 1.4$ & $4.6-8.3$ \\
\hline Boys & 6 & $23.8 \pm 3.8$ & $20.8-29.3$ & $1.02 \pm 0.18$ & $0.90-1.30$ & $5.01 \pm 0.91$ & $4.42-6.36$ & $5.8 \pm 1.0$ & $5.3-7.3$ \\
\hline Combined & 10 & $27.7 \pm 5.3$ & $20.8-36.6$ & $1.23 \pm 0.42$ & $0.83-1.60$ & $6.10 \pm 1.14$ & $4.08-7.82$ & $6.9 \pm 1.2$ & $4.2-8.7$ \\
\hline \multicolumn{10}{|c|}{$2.31-2.80 \mathrm{~m} \cdot \mathrm{s}^{-1}$} \\
\hline Girls & 5 & $31.0 \pm 8.4$ & $19.3-39.2$ & $1.14 \pm 0.28$ & $0.83-1.52$ & $5.56 \pm 1.39$ & $4.08-7.44$ & $6.2 \pm 1.4$ & $4.2-7.3$ \\
\hline Boys & 4 & $30.0 \pm 2.7$ & $27.1-34.4$ & $1.33 \pm 0.16$ & $1.21-1.60$ & $6.53 \pm 0.79$ & $5.94-7.82$ & $7.5 \pm 0.8$ & $6.9-8.7$ \\
\hline Combined & 9 & $30.6 \pm 5.5$ & $19.3-39.2$ & $1.24 \pm 0.23$ & $0.83-1.60$ & $6.10 \pm 1.14$ & $4.08-7.82$ & $6.9 \pm 1.2$ & $4.2-8.7$ \\
\hline \multicolumn{10}{|c|}{$2.81-3.30 \mathrm{~m} \cdot \mathrm{s}^{-1}$} \\
\hline Girls & 1 & 44.7 & - & 1.65 & - & 8.10 & - & 9.1 & - \\
\hline Boys & 2 & $40.9 \pm 1.5$ & $29.8-42.0$ & $1.51 \pm 0.07$ & $1.46-1.55$ & $7.39 \pm 0.32$ & $7.16-7.61$ & $9.1 \pm 0.1$ & $9.1-9.2$ \\
\hline Combined & 3 & $42.2 \pm 2.4$ & $39.8-44.7$ & $1.56 \pm 0.10$ & $1.46-1.65$ & $7.62 \pm 0.47$ & $7.16-8.10$ & $9.1 \pm 0.1$ & $9.1-9.2$ \\
\hline \multicolumn{10}{|c|}{ Free-choice games } \\
\hline Girls & 10 & $25.7 \pm 5.1$ & $18.2-34.0$ & $1.05 \pm 0.18$ & $0.81-1.37$ & $5.16 \pm 0.86$ & $3.95-6.72$ & $6.1 \pm 1.1$ & $4.5-8.0$ \\
\hline Boys & 11 & $26.7 \pm 6.9$ & $14.5-36.0$ & $1.08 \pm 0.23$ & $0.63-1.49$ & $5.27 \pm 1.11$ & $3.06-7.32$ & $5.6 \pm 1.1$ & $3.2-7.0$ \\
\hline Combined & 21 & $26.2 \pm 6.0$ & $14.5-36.0$ & $1.06 \pm 0.20$ & $0.63-1.49$ & $5.22 \pm 0.98$ & $3.06-7.32$ & $5.8 \pm 1.1$ & $\begin{array}{l}3.2-8.0 \\
\text { (continued) }\end{array}$ \\
\hline
\end{tabular}


Table 3 (continued)

\begin{tabular}{|c|c|c|c|c|c|c|c|c|c|}
\hline & \multirow[b]{2}{*}{$\mathbf{n}$} & \multicolumn{2}{|c|}{ O uptake $\left(\mathrm{ml} \cdot \mathrm{kg}^{-1} \cdot \mathrm{min}^{-1}\right)$} & \multicolumn{2}{|c|}{$\mathrm{O}_{2}$ uptake $\left(1 \cdot \mathrm{min}^{-1}\right)$} & \multicolumn{2}{|c|}{$\mathrm{EE}\left(\mathrm{kcal} \cdot \mathrm{min}^{-1}\right)$} & \multicolumn{2}{|c|}{ EE (Child METs) } \\
\hline & & Mean \pm SD & Min-Max & Mean \pm SD & Min-Max & Mean \pm SD & Min-Max & Mean \pm SD & Min-Max \\
\hline \multicolumn{10}{|c|}{ Playground games } \\
\hline Girls & 10 & $24.2 \pm 4.3$ & $19.0-32.8$ & $0.99 \pm 0.15$ & $0.84-1.28$ & $4.89 \pm 0.74$ & $4.11-6.28$ & $5.7 \pm 0.9$ & $4.7-7.6$ \\
\hline Boys & 11 & $23.7 \pm 5.2$ & $15.1-31.9$ & $0.97 \pm 0.24$ & $0.65-1.41$ & $4.78 \pm 1.21$ & $3.20-6.88$ & $5.0 \pm 1.0$ & $3.3-6.5$ \\
\hline Combined & 21 & $23.9 \pm 4.7$ & $15.1-32.8$ & $0.98 \pm 0.20$ & $0.65-1.41$ & $4.82 \pm 0.99$ & $3.20-6.88$ & $5.4 \pm 1.0$ & $3.3-7.6$ \\
\hline \multicolumn{10}{|l|}{ Hopscotch } \\
\hline Girls & 10 & $22.0 \pm 4.5$ & $15.5-32.0$ & $0.90 \pm 0.17$ & $0.72-1.25$ & $4.42 \pm 0.81$ & $3.53-6.11$ & $5.2 \pm 1.0$ & $3.9-7.4$ \\
\hline Boys & 10 & $21.1 \pm 5.2$ & $13.8-28.4$ & $0.87 \pm 0.18$ & $0.60-1.15$ & $4.22 \pm 0.90$ & $2.71-5.62$ & $4.4 \pm 0.8$ & $3.0-5.3$ \\
\hline Combined & 20 & $21.5 \pm 4.7$ & $13.8-32.0$ & $0.88 \pm 0.17$ & $0.60-1.25$ & $4.32 \pm 0.84$ & $2.71-6.11$ & $4.8 \pm 1.0$ & $3.0-7.4$ \\
\hline \multicolumn{10}{|l|}{ Frisbee } \\
\hline Girls & 10 & $25.3 \pm 3.7$ & $19.3-30.0$ & $1.04 \pm 0.15$ & $0.78-1.23$ & $5.09 \pm 0.71$ & $3.80-6.02$ & $6.0 \pm 0.8$ & $4.5-7.1$ \\
\hline Boys & 10 & $25.9 \pm 5.7$ & $14.6-36.0$ & $1.08 \pm 0.31$ & $0.63-1.60$ & $5.29 \pm 1.52$ & $3.08-7.85$ & $5.5 \pm 1.1$ & $3.2-7.0$ \\
\hline Combined & 20 & $25.6 \pm 4.7$ & $14.6-36.0$ & $1.06 \pm 0.24$ & $0.63-1.60$ & $5.19 \pm 1.16$ & $3.08-7.85$ & $5.7 \pm 1.0$ & $3.2-7.1$ \\
\hline \multicolumn{10}{|l|}{ Reaction ball } \\
\hline Girls & 10 & $26.8 \pm 4.9$ & $20.2-37.1$ & $1.10 \pm 0.17$ & $0.89-1.45$ & $5.38 \pm 0.82$ & $4.35-7.10$ & $6.4 \pm 1.0$ & $5.2-8.6$ \\
\hline Boys & 10 & $24.0 \pm 6.4$ & $13.0-33.0$ & $0.97 \pm 0.22$ & $0.64-1.28$ & $4.77 \pm 1.07$ & $3.14-6.27$ & $5.0 \pm 1.1$ & $3.5-6.7$ \\
\hline Combined & 20 & $25.4 \pm 4.7$ & $13.0-37.1$ & $1.04 \pm 0.20$ & $0.64-1.45$ & $5.08 \pm 0.98$ & $3.14-7.10$ & $5.7 \pm 1.2$ & $3.5-8.6$ \\
\hline
\end{tabular}

Note. $\mathrm{ml} \cdot \mathrm{kg}^{-1} \cdot \mathrm{min}^{-1}=$ milliliters (of oxygen) per kilogram body weight; $1 \cdot \mathrm{min}^{-1}=$ liters (of oxygen) per minute.

playground games for children, which on average had an average energy cost of $\sim 5.5$ METs.

There has been some recent debate about the classification of sedentary behavior in children, and whether a threshold of $\leq 1.5$ or $\leq 2$ METs should be used. ${ }^{10}$ On average, both drawing and DVD watching had energy costs that were consistent with the $\leq 1.5 \mathrm{MET}$ threshold, though there was some variability in individual values, and the current values within the youth compendium. ${ }^{1}$ It is postulated that reaching for different materials provided will have resulted in small body movements equivalent to sit-stand transitions, which may have increased the energy cost of the activity. ${ }^{11}$

This study used a field-based protocol to examine the energy cost of children's free-living behaviors. These data contribute to the scarcity of information concerning children's EE during play.

\section{Acknowledgments}

The authors would like to thank all of the children who participated in the study and Kate Eccles for her help during data collection. This work was supported by an Institute for Health Research award.

\section{References}

1. Ridley K, Ainsworth BE, Olds TS. Development of a compendium of energy expenditures for youth. Int J Behav Nutr Phys Act. 2008;5:45. PubMed doi:10.1186/1479-5868-5-45

2. Ainsworth BE, Haskell WL, Whitt MC, et al. Compendium of physical activities: an update of activity codes and MET intensities. Med Sci Sports Exerc. 2000;32(9):S498-S516. PubMed doi:10.1097/00005768-200009001-00009
3. Nilsson A, Brage S, Riddoch C, et al. Comparison of equations for predicting energy expenditure from accelerometer counts in children. Scand J Med Sci Sports. 2008;18(5):643-650. PubMed doi:10.1111/ j.1600-0838.2007.00694.x

4. Stanley RM, Ridley K, Olds TS. The type and prevalence of activities performed by Australian children during the lunchtime and after school periods. J Sci Med Sport. 2011;14(3):227-232. PubMed doi:10.1016/j. jsams.2010.10.461

5. Rowland TW. Developmental Exercise Physiology. Champaign, IL: Human Kinetics; 1995.

6. McArdle WD, Katch FI, Katch VL. Exercise Physiology: Nutrition, Energy, and Human Performance. 7th ed. Philadelphia: Lippincott Williams \& Wilkins; 2010.

7. Schofield WN. Predicting basal metabolic rate, new standards and review of previous work. Hum Nutr Clin Nutr. 1985;39(Suppl 1):5-41. PubMed

8. Trost SG, Loprinzi PD, Moore R, Pfeiffer KA. Comparison of accelerometer cut-points for predicting activity intensity in youth. Med Sci Sports Exerc. 2011;43(7):1360-1368. PubMed doi:10.1249/ MSS.0b013e318206476e

9. Department of Health. Everybody Active, Every Day. An Evidencebased Approach to Physical Activity. London: Department of Health; 2014.

10. Saint-Maurice PF, Kim Y, Welk GJ, Gaesser GA. Kids are not little adults: what MET threshold captures sedentary behavior in children? Eur J Appl Physiol. 2016;116(1):29-38. PubMed

11. Júdice PB, Hamilton MT, Sardinha LB, Zderic TW, Silva AM. What is the metabolic and energy cost of sitting, standing and sit/stand transitions? Eur J Appl Physiol. 2016;116(2):263-73. PubMed 